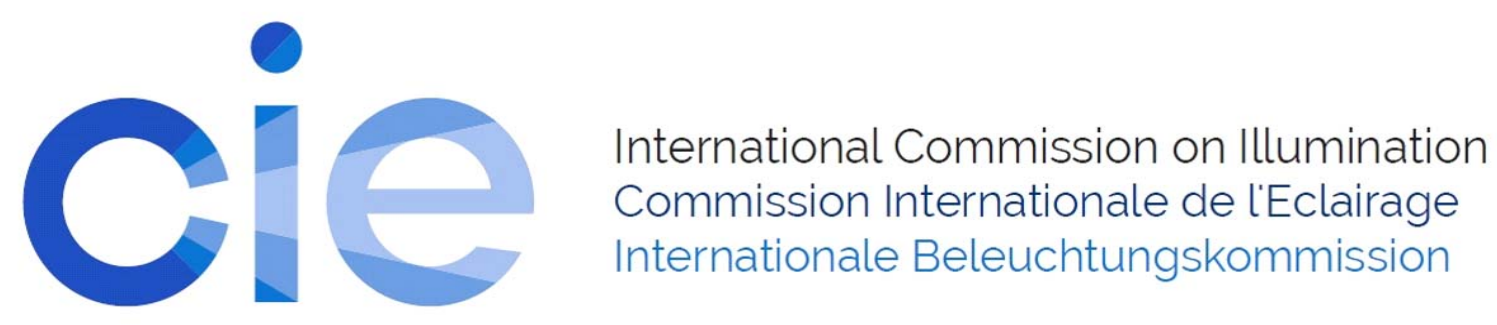

OP71

PROPOSAL OF A NEW WHITENESS FORMULA BASED ON CAM16-UCS

\author{
Yuzhao Wang et al.
}

DOI 10.25039/x46.2019.OP71

from

CIE x046:2019

Proceedings

of the

29th CIE SESSION

Washington D.C., USA, June 14 - 22, 2019

(DOI 10.25039/x46.2019)

The paper has been presented at the 29th CIE Session, Washington D.C., USA, June 14-22, 2019. It has not been peer-reviewed by CIE.

(C) CIE 2019

All rights reserved. Unless otherwise specified, no part of this publication may be reproduced or utilized in any form or by any means, electronic or mechanical, including photocopying and microfilm, without permission in writing from CIE Central Bureau at the address below. Any mention of organizations or products does not imply endorsement by the CIE.

This paper is made available open access for individual use. However, in all other cases all rights are reserved unless explicit permission is sought from and given by the CIE.

CIE Central Bureau

Babenbergerstrasse 9

A-1010 Vienna

Austria

Tel.: +4317143187

e-mail: ciecb@cie.co.at

www.cie.co.at 


\title{
PROPOSAL OF A NEW WHITENESS FORMULA BASED ON CAM16-UCS
}

\author{
Y. Wang ${ }^{1}$, X. Lv ${ }^{1}$, M. Wei ${ }^{2}$, M.R. Luo ${ }^{1,3}$ \\ 1 State Key Laboratory of Modern Optical Instrumentation, Zhejiang University, Hangzhou, \\ CHINA \\ 2 The Hong Kong Polytechnic University, Kowloon, HONG KONG \\ ${ }^{3}$ School of Design, University of Leeds, Leeds LS2 9JT, UNITED KINGDOM \\ m.r.luo@zju.edu.cn
}

DOI 10.25039/x46.2019.OP71

\begin{abstract}
The CIE whiteness formula ( $\left.W_{\left.J^{\prime} a^{\prime} b^{\prime}\right)}\right)$ has been known to have limitations in two aspects. First is the defined boundary to be too restricted and second the formula is only defined under CIE D65 illuminant. This paper described a new whiteness formula based on CAM16-UCS. It has a boundary to define the white region. The formula gave a good prediction to the available data.
\end{abstract}

Keywords: Whiteness, Boundary, CAM16-UCS

\section{Motivation, specific objective}

CIE whiteness formula includes two parts: a limit to define white colour boundary and a formula to calculate the whiteness value for the surface colors containing fluorescent whitening agent (FWAs). The formula has been used for 32 years. It has been realized to have a too constrained boundary and can only be used under CIE D65 illuminant. A CIE TC (1-95 The validity of the CIE whiteness and tint equations) was established to overcome the above problems. This paper describes a new whiteness evaluation system including a white boundary and a whiteness formula based on CAM16-UCS. It is promising that both white boundary and formula gave accurate prediction to the available datasets. Most importantly, they are based on a model of colour vision, which can accurately transform stimulus' chromaticity from all illuminants to that of SE illuminant, and has a visually uniform colour space to define the white boundary and to calculate colour difference.

\section{Methods}

Different datasets were generated for assessing whiteness or white perception. The former data can be used to derive or test existing whiteness formulae. The latter can be used to define the white zone. The most reliable data are used in the present study. A brief description of each dataset is given below.

\subsection{Dataset for defining white boundary}

The dataset was based on the experiment carried out at the Zhejiang University [Wei et al. , 2017]. Twelve observers participated in the experiment. This experiment was performed under a spectrum tunable LED system, LEDCube supplied by the Thouslite. There were 3 different illuminants for CCTs at $3000 \mathrm{~K}, 4000 \mathrm{~K}$, and $6500 \mathrm{~K}$. Each had two UV levels. One was no UV content. The other was adjusted to have $\mathrm{W}_{\mathrm{CIE}}$ values of the samples to be agreed with those measured by a calibrated Datacolor SF600 spectrophotometer using surface standards such as Hohenstein, AATCC, etc. Fifty-five testing samples were measured by a JETI Specbos 1211 tele-spectroradiometer under the $6500 \mathrm{~K}$ lighting condition in the viewing booth. The same UV level was applied to the other two CCT lightings. Each sample was placed in the centre of the mid-grey floor in the viewing booth. After 3 minute's adaptation under each lighting, the observer was asked to report a judgement based on a visual scale from -3 to 3 , where -3 and 3 mean 'no trace of white' and 'pure white', respectively. In total, there were 3 CCTs $\times 55$ samples $\times 12$ observers $\times 2$ UV levels $=3,960$ observations. 


\subsection{Dataset for scaling whiteness (neutrality)}

Two datasets were collected to derive the new whiteness formula. Wei et al. [2018] performed the experiment at the Hong Kong Polytechnic university. A double-cabinet using two LEDCube systems was used: the reference cabinet to illuminant 4 reference samples and the other to illuminate 8 test samples having different contents of FWAs. The test cabinet made 6 light settings, 3 CCTs $(3000,4000,5000 \mathrm{~K})$ and 2 UV levels. Fifteen observers did the experiment to scale the whiteness of each test sample in the test cabinet against the 4 reference samples in the reference cabinet. In total, 720 assessments were made, i.e. 8 samples $\times 3$ CCTs $\times 2$ UV levels $\times 15$ observers.

The second dataset was produced at the Zhejiang University and the results were not published yet. The lighting system was the same as the o described above. Four different CCTs (3000K, $4000 \mathrm{~K}, 5000 \mathrm{~K}$, and $6500 \mathrm{~K}$ ) were studied. The UV content was set the same as described above. Twenty-one nominally white samples with different amounts of FWAs were used in this experiment. 20 observers were asked to look at a reference sample under $6500 \mathrm{~K}$, which was assigned to have a whiteness value of 100 and remember its whiteness appearance. Then without looking at the reference sample, the observer evaluated the whiteness of the test samples one by one under a test illuminant and gave a whiteness value of the test sample by comparing its appearance to the memorized white appearance of the reference sample. A higher score means a whiter appearance. In total, there were 4 CCTs $\times 21$ samples $\times 20$ observers $=1680$ observations. This dataset was used as a training set to develop the new model (see later).

\section{The new whiteness formula}

A new whiteness formula, named white J'a'b' $\left(W_{\left.J^{\prime}{ }^{\prime}{ }^{\prime} b^{\prime}\right)}\right)$ was derived using the datasets introduced in the last section.

$$
W_{J^{\prime} a^{\prime} b^{\prime}}=J^{\prime}+0.295\left(-0.81-a^{\prime}\right)+4.135\left(-2.58-b^{\prime}\right)
$$

where $J^{\prime}, a^{\prime}, b^{\prime}$ are the colour coordinates of the test stimulus in CAM16-UCS within the colour boundary defined in equation (2), and $[-0.81,-2.58]$ are the $a^{\prime} b^{\prime}$ coordinates for the neutral white point. A colour under all illuminants should first be transformed to J'a'b' coordinates with the incomplete adaptation factors $(D)$ of $0.72,0.752,0.772,1.0$ for $3000,4000,5000$ and $6500 \mathrm{~K}$ respectively. Note the reference illuminant for J'a'b' is equal energy illuminant (SE).

For calculating $W_{J^{\prime}} a^{\prime} b^{\prime}$, all colours should be within a zone including all the white colours. The zone was derived by an ellipsoid from the Wei et al dataset.

$$
p=g_{11} J^{\prime 2}+g_{22} a^{\prime 2}+g_{33} b^{\prime 2}+g_{12} J^{\prime} a^{\prime}+g_{13} J^{\prime} b^{\prime}+g_{23} a^{\prime} b^{\prime}+g_{1} J^{\prime}+g_{2} a^{\prime}+g_{3} b^{\prime}+g_{0}
$$

where $p$ is the probability of the test colour being considered as 'none white' and J', a', b' values defined the test colour in CAM16-UCS coordinates. The coefficients in equation (2) are given in Table 1.

Table 1 - The coefficients in equation (2)

\begin{tabular}{|c|c|c|c|c|c|c|c|c|c|}
\hline $\mathrm{g}_{11}$ & $\mathrm{~g}_{22}$ & $\mathrm{~g}_{33}$ & $\mathrm{~g}_{12}$ & $\mathrm{~g}_{13}$ & $\mathrm{~g}_{23}$ & $\mathrm{~g}_{1}$ & $\mathrm{~g}_{2}$ & $\mathrm{~g}_{3}$ & $\mathrm{~g}_{0}$ \\
\hline-2.989 & -1.784 & - & 0.7606 & 0.7701 & - & 565.1 & -76.44 & -76.31 & -26606 \\
\hline
\end{tabular}

When $p$ is larger than 0.5 , the stimulus is located inside the ellipsoid. Otherwise, it is considered as a non-white colour. The equation can be expressed in terms of ellipsoid parameters, i.e. ellipse size $(A)$, ellipse shape $(A / B)$ and ellipse orientation (q) in a'b', J'a' or J'b' plane as given in Table 2. The ellipse is also plotted in Figure 1.

Table 2 - The ellipse parameters in a'b' plane

\begin{tabular}{|c|c|c|c|}
\hline $\mathrm{A}$ & $\mathrm{B}$ & $\mathrm{A} / \mathrm{B}$ & $\theta$ \\
\hline 11.3 & 5.8 & 1.95 & $105^{\circ}$ \\
\hline
\end{tabular}




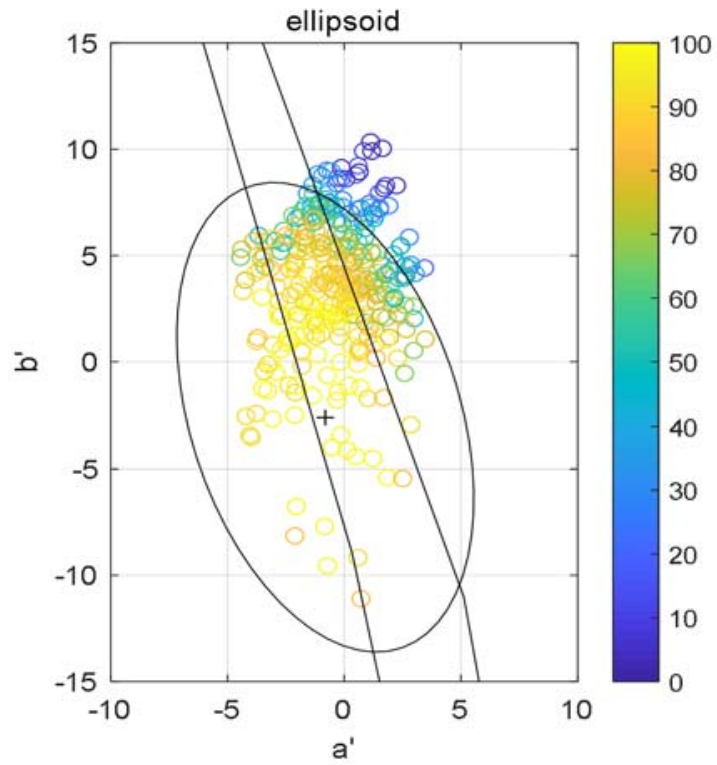

Figure 1 - The white perception data plotted in CAM16-UCS a'b' plane together with the boundaries defined by the ellipsoid (equation 2) and by the tint limit of CIEw.

The boundary of $W_{C I E}$ is also plotted in Figure 1. It can be seen that the CIEw's boundary is much smaller than that defined the ellipsoid (equation (2)). The whiteness formula proposed can predict the visual results well to the two experimental datasets as shown in Figures 2a and $2 \mathrm{~b}$ for the HK and ZJU data respectively, having correlation coefficient values of 0.94 and 0.74 respectively.

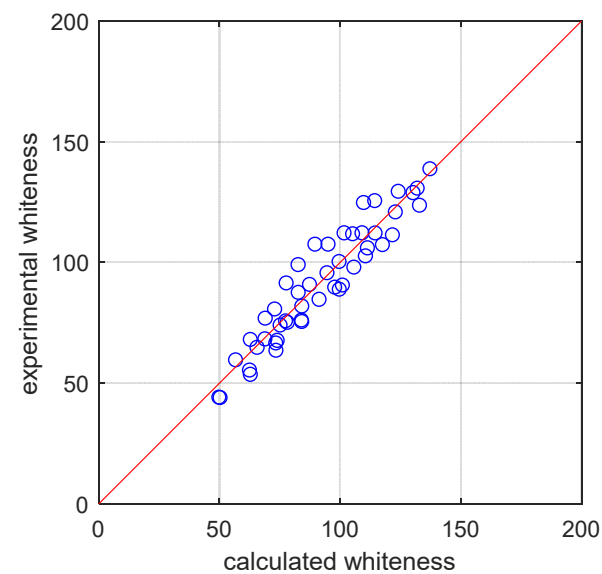

(a) Hong Kong Poly data $(r=0.94)$

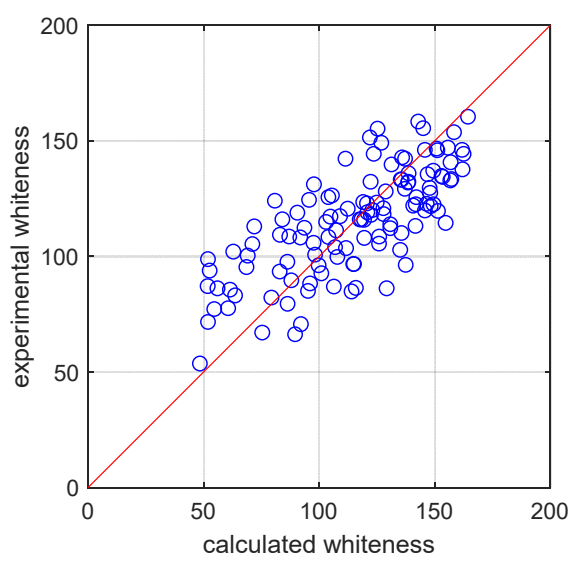

(b) ZJU data $(r=0.74)$

Figure 2 - The visual results plotted against the predictions by the new formula

Overall, the major advantage of the proposed whiteness formula is the use of colour appearance model, CAM16-UCS. It transforms the colours from different illuminants to SE. The white boundary is defined by an ellipsoid. It overcomes the drawbacks of CIEw, having two limits in $\mathrm{Y}$, and tint (red-green), without considering yellow-blue direction in visually non-uniform $\mathrm{xy}$ chromaticity diagram. The aims of TC1-95 are met.

\section{Conclusions}

A new whiteness evaluation system is proposed including a whiteness formula $\left(W_{J^{\prime} a^{\prime} b^{\prime}}\right)$ and a white zone was developed. Their special features are given below.

- The whiteness formula was developed based on the state of the art colour vision model, CAM16-UCS with the incomplete adaptation factors $(D)$ of $0.72,0.752,0.772,1.0$ for 
$3000,4000,5000$ and $6500 \mathrm{~K}$ respectively. CAM16-UCS includes a robust chromatic adaptation transform and a visually uniform colour space.

- The new white zone is clearly defined by a 3D ellipsoid. The current white zone in CIEW had shortage to use limits on $\mathrm{Y}$ and red-green tint directions, without considering yellowblue direction in xy chromaticity diagram.

- The new whiteness formula performs an automatic chromatic adaptation transform to SE illuminant and CAM16-UCS is much more visually uniform than $x-y$ chromaticity diagram.

\section{Acknowledgment}

The work is supported by National Natural Science Foundation of China (Grant number: 61775190)

\section{References}

M. Wei, S. N. Ma, Y. Wang, M. R. Luo. 2017 Evaluation of whiteness formulas for FWA and non-FWA whites. Journal of the Optical Society of Am. A. 1;34(4):640-647.

Wei, S. Chen, H. Huang, M. R. Luo 2018, Development of a whiteness formula for surface colors under an arbitrary light source, Opitcs Express 26(2018)18171-18181. 